05

\title{
Исследование прочностных характеристик алюминиевого сплава 1230 при растяжении в квазистатическом и динамическом диапазонах параметров нагружения
}

\author{
(C) А.Д. Евстифеев, ${ }^{1}$ Г.А. Волков, ${ }^{1,2}$ А.А. Чеврычкина, ${ }^{1,2}$ Ю.В. Петров ${ }^{1,2}$ \\ ${ }^{1}$ Санкт-Петербургский государственный университет, \\ 199034 Санкт-Петербург, Россия \\ ${ }^{2}$ Институт проблем машиноведения РАН, \\ 199178 Санкт-Петербург, Россия \\ e-mail: ad.evstifeev@gmail.com
}

Поступило в Редакцию 17 февраля 2018 г.

В окончательной редакции 18 октября 2018 г.

Принято к публикации 19 ноября 2018 г.

\begin{abstract}
Представлены результаты экспериментального и теоретического исследования прочностных характеристик алюминиевого сплава 1230 при растяжении в квазистатическом и динамическом диапазонах параметров нагружения. С использованием структурно-временного подхода и Sign-Perturbed Sums-алгоритма выполнена оценка возможности повышения скорости деформации образца за счет уменьшения его геометрических размеров.
\end{abstract}

DOI: $10.21883 /$ JTF.2019.05.47466.72-18

\section{Введение}

Стандартные испытания конструкционных материалов при применении в промышленности включают в себя испытания на сжатие, растяжение, изгиб, твердость, реже усталостные испытания. Это позволяет обеспечить выполнение действующих норм по качеству выпускаемого изделия. В случае же с применением материалов в условиях, значительно отличающихся от квазистатических нагрузок, требуются дополнительные испытания и расчетные исследования. Как правило, это связано с изучением свойств материалов при высокоскоростных динамических нагрузках, свойственных не только авиационной, космической и военной отрасли, но и гражданскому строительству в сейсмически активных регионах.

Работы в этом направлении ведутся, начиная со второй половины XX столетия. Было сформировано отдельное направление науки - механика разрушений. При этом появление большого количества экспериментальных и теоретических работ в этом направлении не решило все поставленные задачи. В настоящее время отсутствует общепринятая стандартизированная методика тестирования с последующим анализом полученных данных, которая бы позволяла адекватно оценивать поведение материалов, подвергающихся высокоскоростным импульсным воздействиям.

Основная экспериментальная схема, используемая для тестирования материалов в условиях динамических нагрузок со скоростями деформации от $10^{2}$ до $10^{4} 1 / \mathrm{s}$, основана на методике Кольского с использованием разрезных стержней Гопкинсона [1,2]. Первоначально этот метод был реализован только для одноосных сжимаю- щих нагрузок, но позднее модифицирован для растяжения [3,4], сдвига и других вариаций [5]. При этом в случае эксперимента на растяжение отдельной задачей является реализация экспериментальной схемы и формирование профиля растягивающей нагрузки [6]. Как правило, растягивающее воздействие создается за счет переотражения сжимающего импульса $[3,5]$. Отдельным классом стоят установки, позволяющие реализовывать прямое растяжение $[4,7,8]$.

В настоящей работе была рассмотрена возможность проведения испытаний на растяжение в условиях динамических нагрузок на башенном копре Instron CEAST 9350 с ускорителем. Главными достоинствами используемого оборудования являются реализация прямого одноосного растяжения, а также сертифицированные методики фиксации сигналов и автоматизированная процедура проведения испытаний, что позволяет снизить погрешность эксперимента.

\section{Методика испытаний на растяжение}

Эксперименты на растяжение с низкими скоростями деформации выполнялись на разрывной машине Shimadzu AG-50kNX. Эксперименты на динамическое растяжение выполнялись на башенном копре с ускорителем. Башенный копер Instron CEAST 9350 позволяет проводить эксперименты на растяжение до скорости ударника $24 \mathrm{~m} / \mathrm{s}$ при условии непревышения усилий на датчике величины $2.2 \mathrm{kN}$. В первую очередь были проведены эксперименты с размерами образца, соответствующими ISO 8256 (далее — „Sample I“), с длиной и шириной рабочих частей 10 и $3 \mathrm{~mm}$ соответственно (рис. 1). Все образцы вырезались с использованием 


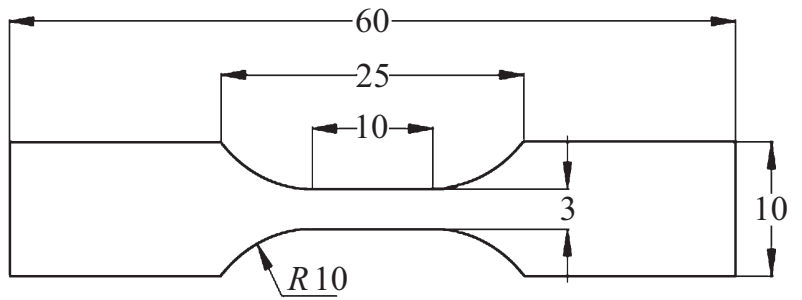

Рис. 1. Форма образца с геометрическими размерами, соответствующими ISO 8256 (Sample I).

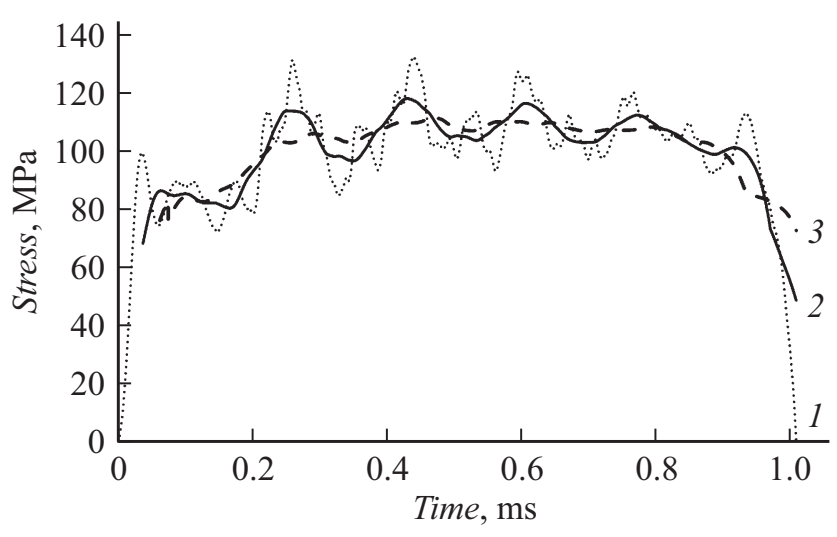

Рис. 2. Диаграммы напряжений при растяжении образца (Sample I) из алюминиевого сплава 1230 в зависимости от количества точек осреднения. Кривая 1 - без осреднения, 2 300 точек осреднения, $3-500$ точек осреднения. Скорость деформации 611 1/s.

электроэрозионного станка ARTA 123 PRO с высокой точностью и доводились на полировальном круге до единых параметров шероховатости. Фиксация сигналов при растяжении образцов на башенном копре происходит в автоматизированном режиме с использованием датчика силы и датчика перемещения ударника.

Эксперименты проводились на алюминиевом сплаве 1230 (ASTM). На рис. 2 кривая 1 - это пример хронограммы инженерных напряжений, полученных путем пересчета усилий, зафиксированных датчиком при растяжении. Сигналы стабильны, но для определения пороговых характеристик материала было предложено проводить операцию осреднения скользящими средними. Интервал осреднения выбирается автоматически в зависимости от длины импульса и не превосходит 30\% его длительности. Это позволяет сгладить возможные случайные выбросы. В качестве примера рассмотрим результаты растяжения образца со скоростью деформации 611 1/s. На рис. 2 представлены диаграммы напряжения в зависимости от количества точек осреднения. В данном случае 1 точка осреднения соответствует исходной диаграмме, 300 точек осреднения соответствуют временному интервалу $75 \mu \mathrm{s}, 500$ точек осреднения соответствуют временному интервалу $125 \mu \mathrm{s}$. В рамках настоящего исследования в качестве пороговой характеристики материала рассматривается среднее значение максимальных напряжений по диаграммам, полученным в ходе осреднения по времени.

Для данных, представленных на рис. 2, среднее значение максимальной величины напряжений равно $120 \mathrm{MPa}$, среднеквадратическое отклонение $7 \mathrm{MPa}$.

Возможности установки Instron позволили достичь скоростей деформации порядка $10^{3} 1 / \mathrm{s}$. Однако для таких режимов испытаний был отмечен повышенный износ отдельных ее частей, потребовавший корректировки методики и снижения скорости ударника. Это было достигнуто за счет уменьшения геометрических размеров образцов. В эксперименте скорость деформации определяется как $\dot{\varepsilon}=v / l$, где $v$ - скорость перемещения, $l$ - длина рабочей части образца. В программном обеспечении установки Instron Ceast 9350 заложена корреляционная формула (1) подсчета изменения скорости ударника вследствие контакта с образцом:

$$
v(t)=v_{0}-\int_{0}^{t} \frac{F(s)-m g}{m} d s,
$$

где $v_{0}$ - скорость ударника в момент соударения, $m$ - масса ударника, $F(t)$ - регистрируемое датчиком усилие в образце в момент времени $t, g$ - коэффициент свободного падения.

$a$

$b$

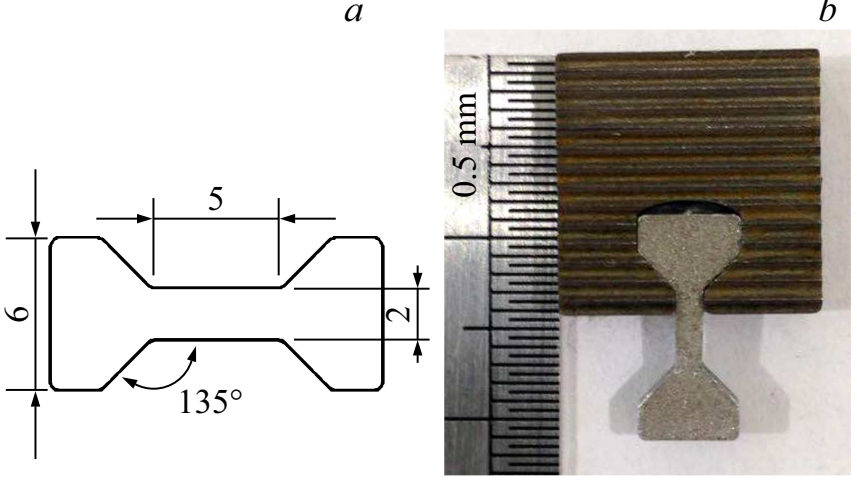

Рис. 3. Геометрические размеры образца (Sample II) с длиной и шириной рабочей части 5 и $2 \mathrm{~mm}$ соответственно, и дополнительной державки.

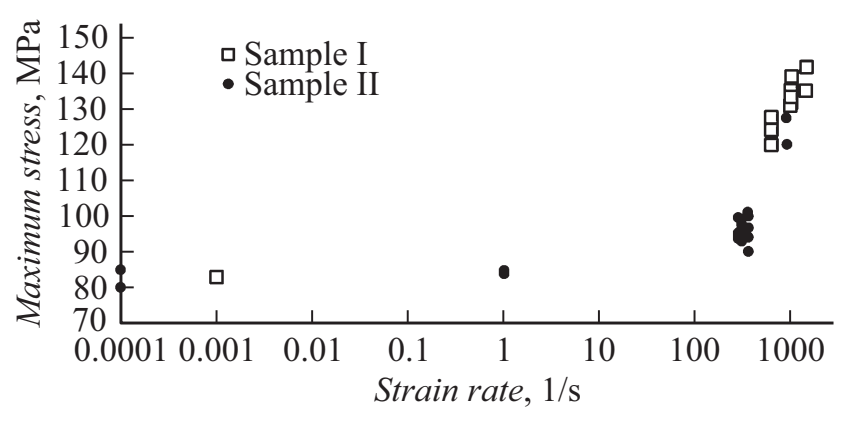

Рис. 4. Зависимость максимальной прочности на растяжение от скорости деформации образцов типов Sample I и Sample II из алюминиевого сплава 1230. 
Вариацией геометрических размеров образцов в соответствии с их прочностными характеристиками можно реализовать условие незначительного отклонения скорости ударника $(<1-3 \%)$ от начальной скорости после столкновения, и как следствие, постоянную скорость деформации.

Для увеличения скорости деформации была выбрана геометрия рабочей части с длиной $5 \mathrm{~mm}$ и шириной $2 \mathrm{~mm}$ (далее - „Sample II“, рис. 3,a). Для установки образцов такого размера потребовалось разработать дополнительный элемент крепления образца в стандартные захваты (рис. $3, b$ ).

Уменьшение длины рабочей части образцов позволило достичь скорости деформации $10^{3} 1 / \mathrm{s}$ при существенно меньших скоростях ударника. На рис. 4 представлены значения прочности на растяжение в зависимости от скорости деформации образцов из алюминиевого сплава 1230 с геометрическими размерами по ISO 8256 и образцов с уменьшенными геометрическим размерами. Исходя из анализа полученных данных, можно отметить нелинейное возрастание прочности с ростом скорости приложения нагрузки.

Полученные результаты экспериментального определения прочностных характеристик материала в статическом и динамическом диапазонах изменения параметров внешнего воздействия показали хорошее соответствие на двух типах образцов в статическом диапазоне внешнего воздействия. Однако точную оценку соответствия результатов из визуального анализа дать не представляется возможным, поскольку с ростом скорости деформации зависимость прочности носит нелинейный характер и становится более чувствительной к нагрузке. Для оценки применимости геометрических размеров образцов уменьшенных размеров воспользуемся критерием инкубационного времени, апробированном на большом количестве экспериментальных данных [9-11].

\section{Анализ экспериментальных данных}

В настоящей работе были выполнены эксперименты по растяжению образцов в условиях квазистатических и динамических нагрузок. В качестве критерия разрушения материала при высокоскоростном нагружении рассмотрим критерий инкубационного времени в виде $[12,13]$ :

$$
\frac{1}{\tau} \int_{t-\tau}^{t} \frac{\sigma(s)}{\sigma_{c}} d s \leq 1,
$$

где $t-$ время, $\sigma$ - приложенное напряжение, $\sigma_{c}-$ статический предел прочности, $\tau$ - инкубационное время разрушения. Константы $\sigma_{c}$ и $\tau$ являются параметрами материала.

Использование экспериментальных данных позволило определить параметры материала для критерия (2) методом наименьших квадратов (МНК) и построить

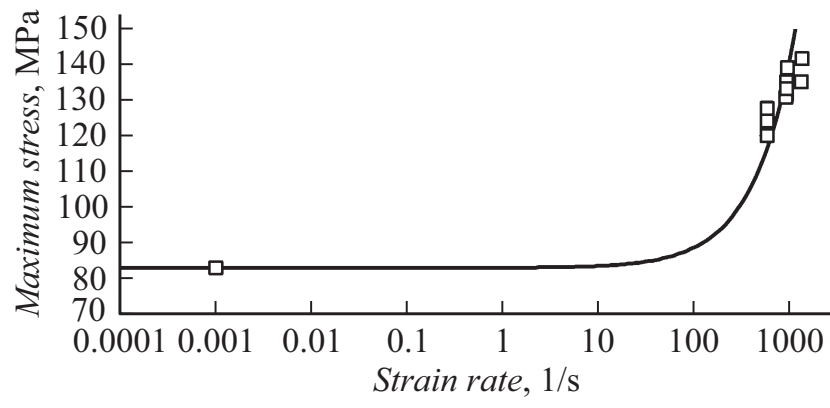

Рис. 5. Расчетная и экспериментальная зависимости максимальной прочности на растяжение от скорости деформации образцов Sample I из алюминиевого сплава 1230.

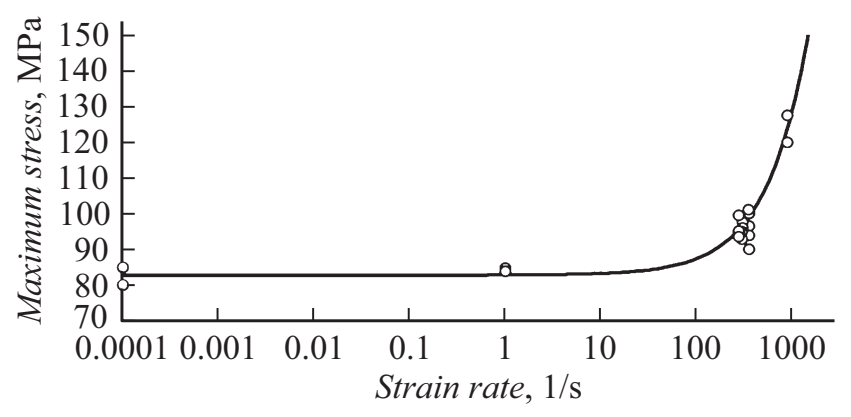

Рис. 6. Расчетная и экспериментальная зависимости максимальной прочности на растяжение от скорости деформации образцов Sample II из алюминиевого сплава 1230.

расчетные зависимости прочности материала от скорости деформации для случая линейно возрастающей по времени нагрузки. Метод МНК обеспечивает точечную оценку [14] и позволяет получать значения, пригодные для дальнейшего анализа. На рис. 5 представлены экспериментальные данные и результаты теоретических расчетов, отражающие зависимость максимального напряжения при растяжении от скорости приложения нагрузки для больших образцов Sample I. Расчетная кривая построена с учетом следующих величин: $E=72 \mathrm{GPa}$, $\tau=1.4 \mu \mathrm{s}, \sigma_{c}=83 \mathrm{MPa}$. На рис. 6 представлены экспериментальные данные и теоретические расчеты для образцов уменьшенного размера Sample II. Расчетная кривая построена с учетом следующих величин: $E=72 \mathrm{GPa}$, $\tau=1.2 \mu \mathrm{s}, \sigma_{c}=83 \mathrm{MPa}$. Дополнительно был произведен расчет и определены параметры материала $E=72 \mathrm{GPa}$, $\tau=1.3 \mu \mathrm{s}, \sigma_{c}=83 \mathrm{MPa} \mathrm{с} \mathrm{использованием} \mathrm{эксперимен-}$ тальных данных, полученных на двух типах образцов.

Анализируя полученные данные, а именно параметр материала $\tau$, отвечающий за динамическую прочность, можно судить о возможности использования образцов двух типов. Параметр инкубационного времени для образцов Sample I и Sample II отличается на 8\% от среднего значения. Причем в рассматриваемом примере среднее значение $\tau$ соответствует параметру, определенному с использованием всех экспериментальных точек. 


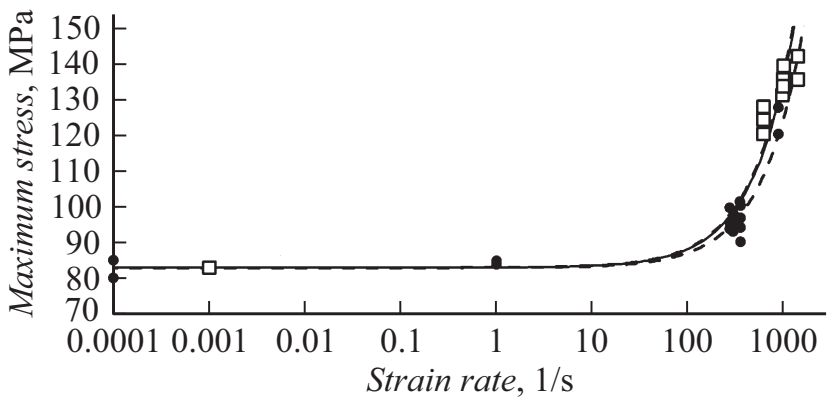

Рис. 7. Расчетная и экспериментальная зависимости максимальной прочности на растяжение от скорости деформации образцов Sample I и Sample II из алюминиевого сплава 1230. Штриховые линии построены по критерию (2) согласно SPS-процедуре для $\tau \in[1.2 ; 1.5]$, сплошная линия построена по критерию (2) согласно МНК для $\tau=1.3 \mu \mathrm{s}$.

Несмотря на хорошую сходимость результатов расчетных исследований с экспериментальными данными, а также близость точечных оценок параметров материалов, полученных по МНК, для инженерных расчетов необходимо знать доверительные интервалы для параметра $\tau$ с достаточно высокой степенью достоверности. Это позволит минимизировать ошибку, связанную с нелинейным поведением зависимости прочности материала от скорости приложения нагрузки. Оптимальным решением может являться SPS (Sign-Perturbed Sums) метод [15], который позволяет строить неасимптотические доверительные интервалы для неизвестного параметра. Данный метод уже успел успешно себя зарекомендовать применительно к задачам по определению инкубационного времени разрушения [16,17]. Принципиальной особенностью данного подхода является его применимость в случае небольшого количества наблюдений, при этом недостаток экспериментальных данных компенсируется проведением дополнительных численных испытаний. Задачу определения значения неизвестного параметра по результатам экспериментов можно рассматривать, как следующую модель наблюдений:

$$
\sigma_{i}^{d}=\varphi\left(\dot{\varepsilon}_{i}, \tau^{*}\right)+v_{i}, \quad i=1 \ldots N,
$$

где $\sigma_{i}^{d}-$ напряжения в момент разрушения, $\varphi\left(\dot{\varepsilon}_{i}, \tau^{*}\right)-$ функция, описывающая скоростную зависимость прочности согласно структурно-временному критерию, $\dot{\varepsilon}_{i}$ - скорость деформации при нагружении, $\tau^{*}-$ истинное значение инкубационного времени, величину которого требуется измерить, $v_{i}-$ случайные помехи. Для расчетов доверительного интервала значений инкубационного времени $\tau$ SPS-методом, требуется выбрать значения основных целочисленных параметров $M$ и $q$, где $M>q>0$, с учетом необходимого уровня достоверности $P=1-\frac{q}{M}$. Затем сгенерировать набор из $N(M-1)$ случайных знаков $\beta_{i j}$ : $\operatorname{Prob}\left\{\beta_{i j}=1\right\}=\operatorname{Prob}\left\{\beta_{i j}=1\right\}=\operatorname{Prob}\left\{\beta_{i j}=-1\right\}=1 / 2$ и составить следующие знако-возмущенные суммы:

$$
H_{0}(\tau)=\sum_{i=1}^{N} \frac{\partial \varphi\left(\dot{\varepsilon}_{i}, \tau\right)}{\partial \tau}\left(\sigma_{i}^{d}-\varphi\left(\dot{\varepsilon}_{i}, \tau\right)\right)
$$

и

$$
H_{j}(\tau)=\sum_{i=1}^{N} \beta_{i j} \frac{\partial \varphi\left(\dot{\varepsilon}_{i}, \tau\right)}{\partial \tau}\left(\sigma_{i}^{d}-\varphi\left(\dot{\varepsilon}_{i}, \tau\right)\right) .
$$

После расстановки $\left|H_{j}(\tau)\right|$ по возрастанию определяется ранг данного значения $\Re(\tau)$, равный порядковому номеру $H_{0}(\tau)$ в упорядочивании $\left\{\left|H_{j}(\tau)\right|\right\}_{j=0}^{M}$. В конечный доверительный интервал попадают только те значения, для которых $\Re(\tau) \leq M-q$.

Полученные с использованием SPS-алгоритма интервалы параметра инкубационного времени разрушения для образцов Sample I, Sample II и их совместного рассмотрения демонстрируют хорошее соответствие с полученными МНК точечными оценками. Для случая 95\% доверительной вероятности были получены следующие интервалы. Для Sample I параметр $\tau \in[1.2 ; 1.7]$, для Sample II параметр $\tau \in[1.1 ; 1.4]$, в случае общего рассмотрения параметр $\tau \in[1.2 ; 1.5]$. Точечные оценки параметра $\tau$ во всех случаях принадлежат найденным интервалам. На рис. 7 представлены экспериментальные данные и расчетные кривые для двух типов образцов.

\section{Заключение}

В работе были исследованы прочностные характеристики алюминиевого сплава в квазистатическом и динамическом режимах нагружения. Переход от квазистатического к высокоскоростному режиму нагружения, помимо увеличения скорости воздействия, дополнительно был достигнут за счет изменения геометрии образца. Первый — в соответствии с ISO 8256 с длиной и шириной рабочей части 10 и $3 \mathrm{~mm}$ соответственно, второй - уменьшенных размеров с длиной и шириной рабочей части 5 и $2 \mathrm{~mm}$ соответственно.

Экспериментально были получены зависимости предельных значений усилия при растяжении от скорости деформации, носящие нелинейный характер. С использованием структурно-временного подхода дано обоснование полученных эффектов как в динамическом, так и в квазистатическом режимах нагружения. Предложен новый метод обработки экспериментальных данных (SPS-метод), позволяющий получить интервальную оценку для истинного значения модельных параметров материала.

С использованием структурно-временного подхода и SPS-метода выполнен сравнительный анализ экспериментальных данных, полученных на двух типах образцов, который показал корреляцию результатов в широком диапазоне изменения параметров нагружения с достаточно высокой степенью достоверности.

Использованный экспериментально-теоретический метод тестирования материала на динамическую 
прочность с применением SPS-метода показал свою применимость и может быть рекомендован для комплексного испытания материалов, планируемых к эксплуатации в условиях динамических нагрузок.

Работа выполнена при финансовой поддержке гранта РНФ (№ 17-79-10145). Экспериментальные исследования проведены с использованием оборудования ресурсного центра Научного парка СПбГУ „Исследование экстремальных состояний материалов и конструкций“ и лаборатории „Механики перспективных массивных наноматериалов для инновационных инженерных приложений ${ }^{6}$.

\section{Список литературы}

[1] Kolsky H. // Proceedings of the Physical Society. Section B. 1949. Vol. 62. N 11. P. 676-700.

[2] Hopkinson B. // Philosophical Transactions of the Royal Society of London. Series A, Containing Papers of a Mathematical or Physical Character. 1914. Vol. 213. P. 437456.

[3] Nicholas T. // Express Mech. 1981. Vol. 21. N 5. P. 177-195.

[4] Staab G.H., Gilat A. // Experimental Mechanics. 1991. Vol. 31. N 3. P. 232-235.

[5] Bragov M., Lomunov A.K. // Int. J. Impact Engng. 1995. Vol. 16. N 2. P. 321-330.

[6] Lindholm U.S., Yeakley L.M. // Experimental Mechanics. 1968. Vol. 8. N 1. P. 1-9.

[7] Cadoni E., Albertini C., Solomos G. // J. Phys. IV. 2006. Vol. 134. P. 647-652.

[8] Mancini E., Sasso M., Rossi M., Chiappini G., Newaz G., Amodio D. // J. Dynamic Behavior Materials. 2015. Vol. 1. N 2. P. 201-213.

[9] Евстифеев А.Д., Груздков А.А., Петров Ю.В. // ЖТФ. 2013. T. 83. Вып. 7. С. 59-63.

[10] Petrov Y., Smirnov I., Evstifeev A., Selyutina N. // Frattura ed Integrità Strutturale. 2013. N 24. P. 112-118.

[11] Evstifeev A., Petrov Y., Bragov A., Konstantinov A. // Procedia Structural Integrity. 2016. N 2. P. 446-451.

[12] Morozov N.F., Petrov Y.V. Dynamics of Fracture. Berlin: Springer, 2000. $99 \mathrm{p}$.

[13] Petrov Y., Morozov N. // Trans. ASME J. Appl. Mech. 1994. Vol. 61. N 3. P. 710-712.

[14] Ljung L. System Identification: Theory for the User. Englewood Cliffs. HJ, Prentice Hall. 1999. 609 p.

[15] Csáji B.C., Campi M.C., Weyer E. // IEEE Transactions on Signal Processing. 2015. Vol. 63. N 1. P. 169-181.

[16] Volkova M.V., Granichin O.N., Volkov G.A., Petrov Yu.V. // Vestnik St. Petersburg University, Mathematics. 2018. Vol. 51. N 1. P. 23-30.

[17] Volkova M.V., Granichin O.N., Petrov Yu.V., Volkov G.A. // Advances in Systems Science and Applications. 2017. Vol. 17. P. 34-41. 